\title{
The ACS LCID project: accurate measurements of the full star formation history in low metallicity, isolated, Local Group dwarf galaxies
}

\author{
Matteo Monelli ${ }^{1} \dagger$ and the LCID team \\ ${ }^{1}$ Instituto de Astrofísica de Canarias, \\ C/ Vía Láctea, 38205, La Laguna, Tenerife, Spain \\ email: monelli@iac.es
}

\begin{abstract}
We present here the latest results of the LCID project (Local Cosmology from Isolated Dwarfs), aimed at recovering the full star formation history (SFH) of six isolated dwarf galaxies of the Local Group (LG). Our method of analysis is based on the IAC-pop code, which derives the SFH of a resolved stellar system by comparing the observed and a model color-magnitude diagram (CMD). We summarize here basic technical issues and the main results concerning our sample of galaxies. We show that LeoA is the only object showing a clear delay in the onset of the major SF event, while all the other galaxies present a dominant component older than 10 Gyrs.
\end{abstract}

Keywords. galaxies: evolution, galaxies: dwarf, (galaxies:) Local Group

\section{Introduction}

The study of nearby resolved galaxies is a powerful tool to shed light on the formation and evolution of galaxies. In particular, the importance of isolated galaxies relies on the fact that they are likely approaching the Local Group (LG) for the first time, and therefore they spent most of their life time free from the influence of giant galaxies. These objects are ideal candidates to study the first stages of their formation and evolution, and can provide key insight in the cold dark matter scenery. Our approach to recover the SFH of resolved stellar systems is based on sophisticated modelling of their CMD. In particular, the most reliable constraints can be derived from the main sequence turn-offs (MSTO, Gallart et al. 1999). Therefore, the galaxies of our sample were selected balancing two opposing requirements. Isolated, and therefore distant, objects were required, but deep and accurate photometry was mandatory to detect the TO stars with good signalto-noise ratio $(>10)$. The final sample includes galaxies of different morphological type: Cetus and Tucana (dSph), LGS3 and Phoenix (transition dIrr/dSph), IC1613 and LeoA (dIrr). With the exception of Phoenix, for which we used WFPC2 data, all of the galaxies were observed with the ACS camera on Hubble Space Telescope (HST). Our method is based on the application of the IAC-star and IAC-pop codes. The latter makes use of a genetic algorithm to derive the star formation rate (SFR) and the chemical evolution law of a stellar system, comparing the observed and a model CMDs. A few technical aspects of the method are discussed (a complete description can be found in Aparicio \& Gallart 2004 and in Aparicio \& Hidalgo 2008).

$\dagger$ Local Cosmology from Isolated Dwarfs, http://www.iac.es/project/LCID 

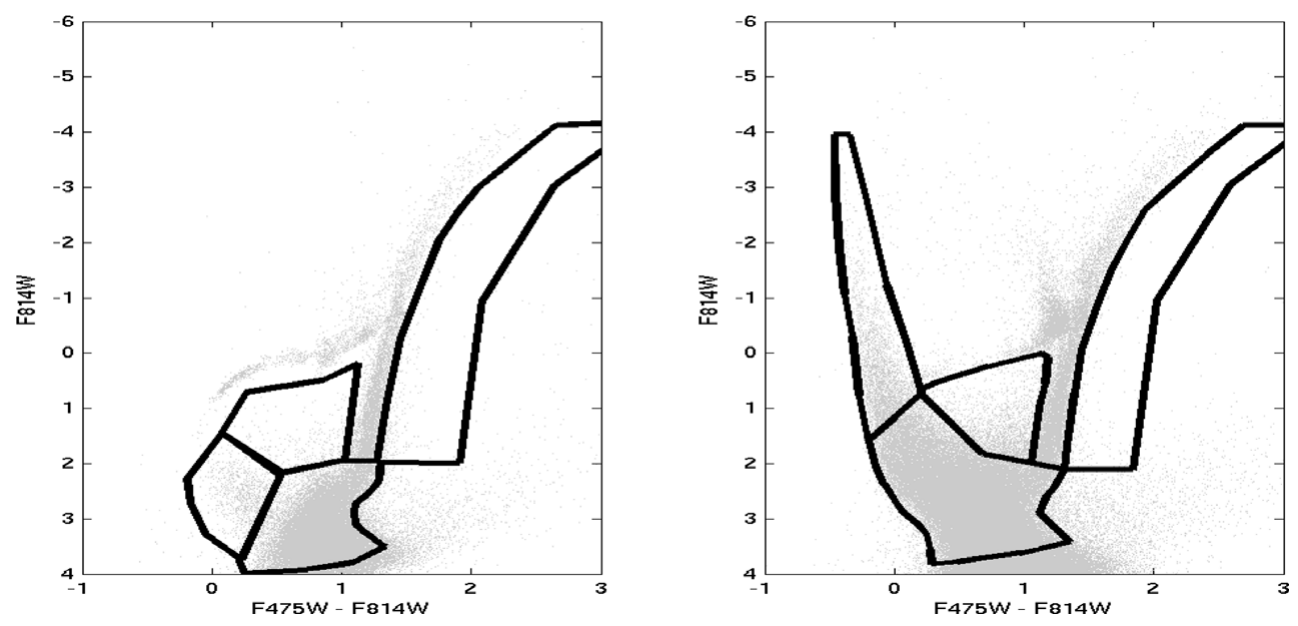

Figure 1. Two examples of parametrization of a CMD: Tucana (left) and IC1613 (right). The four regions define the bundles which are sub-divided into boxes for the star counting. We adopt the finest sampling in the MS and MSTO regions.

\section{The method: IAC-pop}

The basic idea of IAC-pop is that any SFH is mainly characterized by two parameters, the age and the metallicity of the stars. The $\mathrm{SFH}, \Psi(t, Z)$, is therefore a function described as the combination of single populations, defined as the ensembles of stars formed per unit of time and metallicity, and can be expressed as:

$$
\Psi(t, Z)=A \sum_{i} \alpha_{i} \psi_{i}
$$

where $\Psi(t, Z)$ is the global SFH, $\psi_{i}$ are the contributions of the single populations, and $\mathrm{A}$ is the normalization constant. In this representation, the weights $\alpha_{i}$ give a quantitative estimate of the mass of gas transformed into stars at that particular age and with that particular metallicity. From a practical point of view, the single populations are identified using a discrete set of time and metallicity bins. The values adopted in this work are the following: age bins of $1 \mathrm{Gyr}$ in the range 0 to $15 \mathrm{Gyr}$, except the last bin between 13 and 15 Gyr, Z = [0.0001, 0.0003, 0.0005, 0.0007, 0.001, 0.0015, 0.002], being this last value extended to 0.003 in the case of LGS3 and LeoA, and to 0.005 for IC1613.

The model CMD is generated using the IAC-star simulator, and requires a number of different ingredients:

- a set of theoretical stellar evolution libraries. Here we used the BaSTI (Pietrinferni et al. 2004) models, because it covers the full metallicity range expected in the observed galaxies;

- the SFR, which we set constant at any age;

- the metallicity law, Z(t), which we define such that the metallicity of stars of any age is uniformly distributed in the range $0.0001<\mathrm{Z}<0.005$;

- the initial mass function (IMF), taken from Kroupa (2001);

- the binary fraction, $\beta$, and the relative mass distribution $q$, the default values being $\beta=60 \%, q>0.5$.

Other auxiliary but fundamental information is necessary to compare the model with the observations. First, an independent estimate of the distance modulus (DM) and the extinction $\mathrm{E}(\mathrm{B}-\mathrm{V})$ are mandatory to shift the theoretical and observed diagrams in the same magnitude system. Second, the observational errors (crowding, blending, 


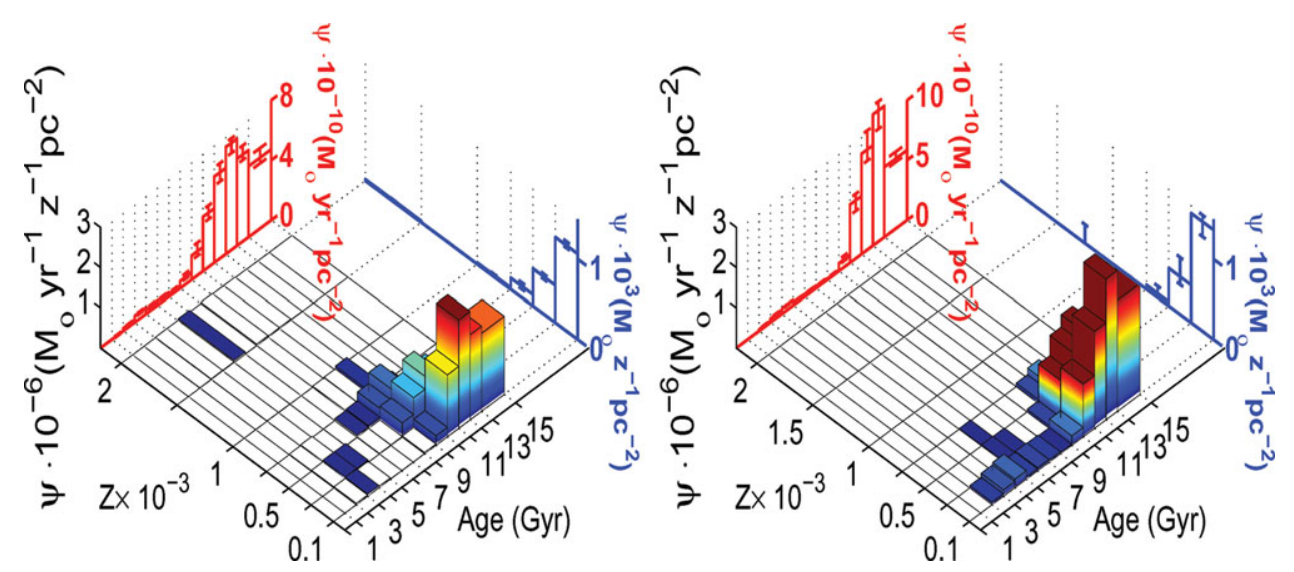

Figure 2. 3-D representation of the SFH of the two dSph galaxies in our sample: Tucana and Cetus.

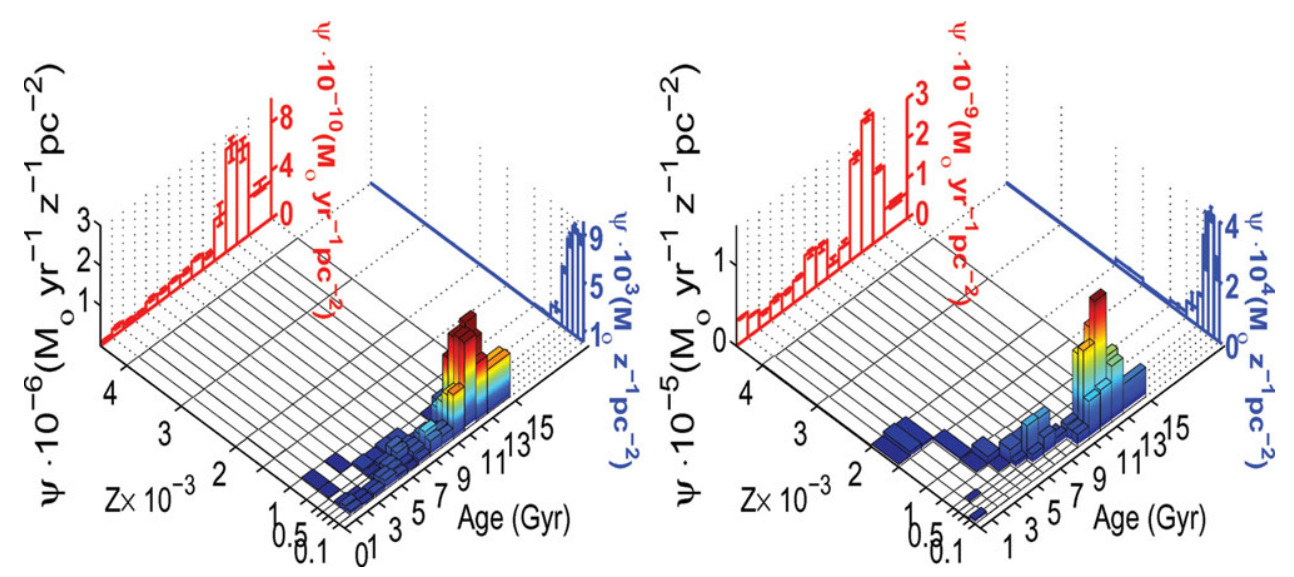

Figure 3. Same as for Fig. 2, but for LGS3 and Phoenix.

incompleteness, photometric errors, ...) have to be properly simulated in the model CMD (Aparicio \& Gallart 1995). This is done through extensive tests adding synthetic stars to the images, and repeating the full process of the photometric analysis. On average, 700,000 synthetic stars were used for each galaxy.

The observable that IAC-pop uses to compare the data with the model is the number of stars, using a $\chi^{2}$ merit function (Mighell 1999). A key point of the method is that the CMDs have to be parametrized in order to perform the star count. This is made by defining a set of boxes covering the CMDs (see Fig. 1). It is important to notice that different features of the CMD are sampled in different ways. Fig. 1 identifies various regions (bundles); each of them is sub-divided into boxes. Extensive tests disclosed that one has to rely the most on the features where most of the information resides, and where the theoretical uncertainities of the models are the smallest. Thefore, we adopt the finest sampling on the MS, the TO and the sub-giant branch regions. Note that we don't use at all the red giant and horizontal branches, given their small temporal resolution and theroretical uncertainities, respectively. 

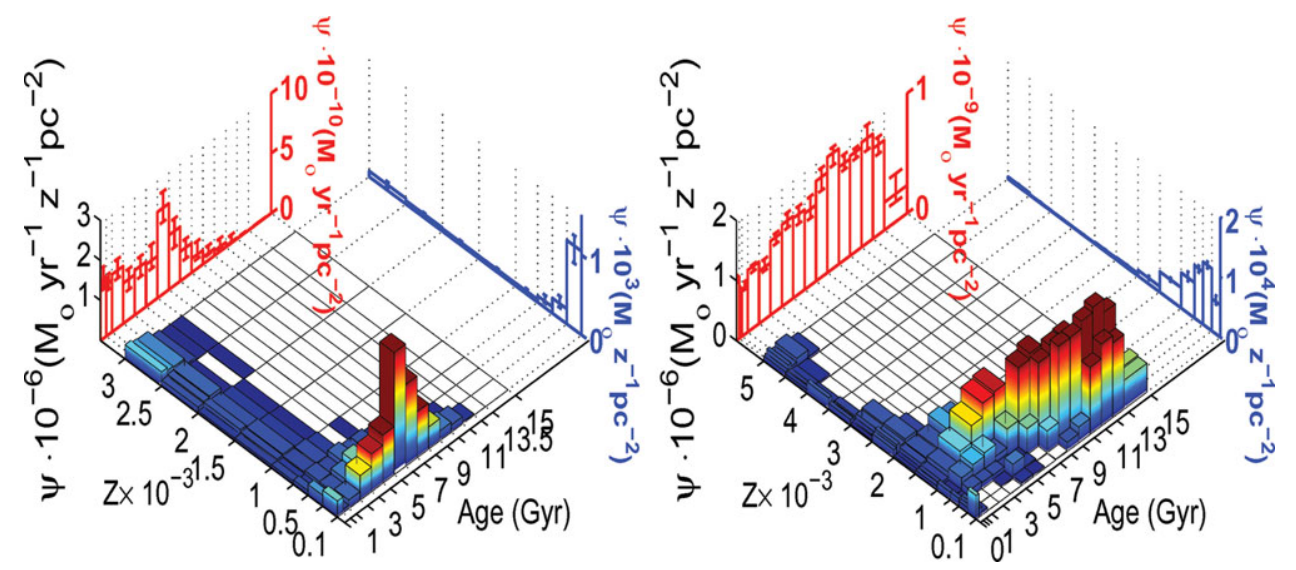

Figure 4. Same as for Fig. 3, but for LeoA and IC1613.
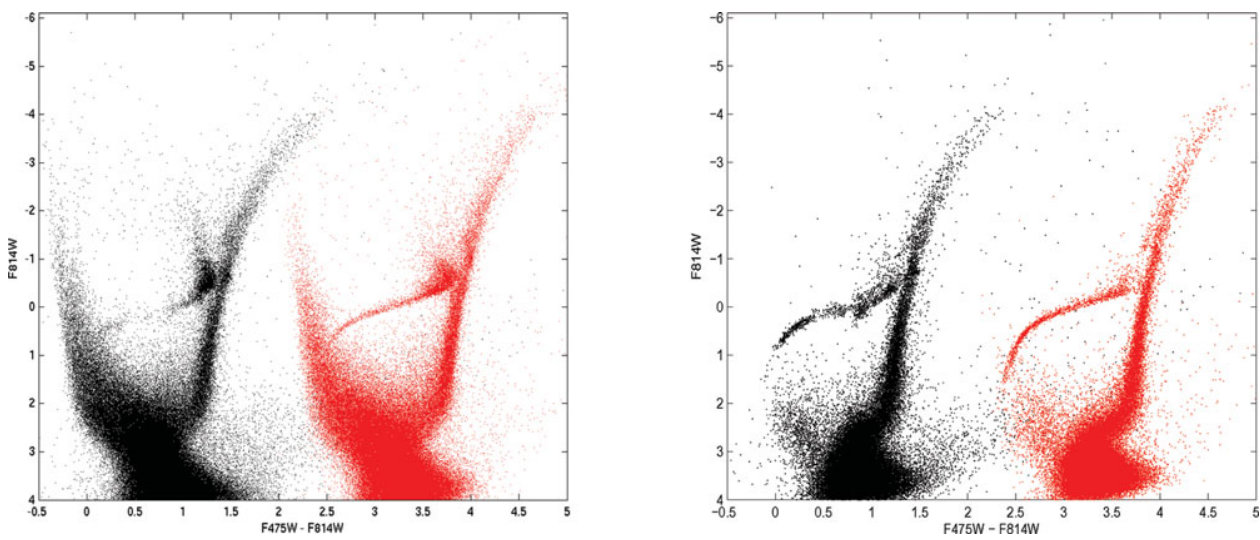

Figure 5. In each panel are shown the observed CMD (left) and the CMD corresponding to the derived SFH. The two galaxies represented are IC1613 and Tucana.

\section{On the solutions and their stability}

Figs. 2 to 4 present the SFH of the six sample galaxies.

i) Cetus and Tucana: these two dSph galaxies are predominantly old and metal-poor systems. The bulk of the SF occurred at old ages, the peak being around 12 Gyrs ago. Both present declining SFR, with negligible star formation at epochs more recent than 10 Gyrs;

ii) LGS3 and Phoenix: the two transition galaxies in our sample present a continuous $\mathrm{SF}$ all over their lifetime. Again, the most important episode of SF occurred at old epochs (>12 Gyr), followed by a steady decline until $\approx 7$ Gyr ago. However, both systems were able to form stars until present ages, with a mild but continuous process.

iii) LeoA and IC1613: LeoA presents the most striking example of delayed SF (Cole et al. 2007). Although the presence of an old population is testified by the presence of RR Lyrae stars (Dolphin et al. 2002), this galaxy has experienced a dominating event of $\mathrm{SF} \approx 6-8$ Gyrs ago. IC1613 seems to show a similar trend, even if to a much less dramatic extent. The right panel of Fig. 4 shows that the SF of this galaxy became more and more intense for ages from $\approx 13$ to $\approx 10$ Gyrs, reached a peak and then started declining.

Fig. 5 shows the CMD corresponding to the solution for IC1613 and Tucana. The overall agreement is satisfactory, and it is interesting to note that the RGB is very well 
reproduced, even if no information at all from this feature has been used to derive these solutions.

To study the uncertainities and the stability of these solutions, we first have to identify the possible sources of error. For the sake of clarity, we can classify them in three groups:

- observational errors: crowding, incompleteness, ...;

- theoretical errors: uncertainties in the stellar evolution models;

- external errors: distance, extinction, binary fraction, IMF, ... .

We assume that the observational errors are properly modelled by our reduction procedure, and a full discussion concerning the use of different stellar evolution libraries (BaSTI and the Padova library, Bertelli et al. 1994) will be presented in a forthcoming paper (Hidalgo et al., in prep.).

Our work-in-progress is mainly focused at analysing the external errors, because this will allow us to estimate the impact of our assumptions on the derived SFH. This is done by changing the input parameters of the model CMD. In particular, we are testing different: i) fraction of binary stars (from 0 to $100 \%)$; ii) values of the distance $( \pm 0.15$ mag); iii) values of the extinction; iv) IMF (changing one or both the IMF indices).

The in-depth analysis and results will be presented in the papers in preparation.

\section{References}

Aparicio, A. \& Gallart, C. 1995, AJ, 110, 2105

Aparicio, A. \& Gallart, C. 2004, AJ, 128, 1465

Aparicio, A. \& Hidalgo, S. 2008, AJ, Submitted

Bertelli, G., Bressan, A., Chiosi, C., Fagotto, F., \& Nasi, E. 1994 A\&AS, 106, 275

Cole, A. A., Skillman, E. D., Tolstoy, E., Gallagher, J. S., III, Aparicio, A., Dolphin, A. E., Gallart, C., Hidalgo, S. L., Saha, A. t, Stetson, P. B., \& Weisz, D. R. 2007 ApJ (Letters), 659,17

Dolphin, A. E., Saha, A., Claver, J., Skillman, E. D., Cole, A. A., Gallagher, J. S., Tolstoy, E., Dohm-Palmer, R. C., \& Mateo, M. 2002 AJ, 123, 3154

Gallart, C., Freedman, W. L., Aparicio, A., Bertelli, G., \& Chiosi, C. 1999, AJ, 1181, 2245

Kroupa, P. 2001, MNRAS, 322, 231

Mighell, K. J., 1999, ApJ, 518, 380

Pietrinferni, A., Cassisi, S., Salaris, M., \& Castelli, F. 2004, ApJ, 612, 168 